\title{
The relative risk of spatial cluster occurrence and spatio- temporal evolution of meningococcal disease in Niger, 2002-2008
}

\author{
Halima B. Maïnassara ${ }^{1}$, Nicolas Molinari ${ }^{2,3}$, Christophe Dematteï ${ }^{2}$, Pascale Fabbro-Peray ${ }^{2,4}$ \\ ${ }^{1}$ Centre de Recherches Médicales et Sanitaires (CERMES)/Résean International des Instituts Pasteur, \\ Niamey, Niger; ${ }^{2}$ Département de Biostatistiques, Epidémiologie, Santé Publique et Information Médicale, \\ CHU de Nîmes, Nimes, France; ${ }^{3}$ UMR729 MISTEA, Montpellier SupAgro, Montpellier, France; ${ }^{4}$ EA2415, \\ Institut Universitaire de Recherche Clinique, Université Montpellier I, Montpellier, France
}

\begin{abstract}
Meningococcal disease is a major public health concern in Sahelian Africa, where over half of the cases reported worldwide occur. In an effort to find annual spatial clusters of meningococcal disease and in order to study their evolution in Niger from January 2002 to June 2008, a prospective study of routine national surveillance data was conducted pertaining to patients with suspected bacterial meningitis. The diagnoses were obtained by analysing patients' cerebrospinal fluid, using polymerase chain reaction or bacteriology. SatScan using Poisson's model was used to calculate the relative risk (RR) of occurrence of spatial clusters. In the 2002-2003 period, 15 spatial clusters of meningococcal meningitis were detected in a total of 3,979 cases with a maximum number of 558 cases per cluster in the south-eastern part of the country $(70.5 \%$ of all cases that year; $R R=7.85 ; \mathrm{P}<0.001)$. Other clusters were found in the following years in approximately the same area as those detected in 2002-2003. These clusters were identified in the southeast, which allowed us to identify high-risk groups in this part of the country. Statistically significant spatio-temporal patterns were found, which should be useful in establishing hypotheses for prospective studies on epidemic tendencies and empirical risk factors in the African meningitis belt.
\end{abstract}

Keywords: meningococcal disease, relative risk, clusters analysis, Niger.

\section{Introduction}

Meningococcal disease is a major public health concern in Sahelian Africa, where over half of the cases reported worldwide occur. Lapeyssonie (1963) described the geographical area named the African meningitis belt (AMB) in which these epidemics occur. In addition to the annual seasonal cases, devastating epidemics arise periodically with a recur-

\footnotetext{
Corresponding author:

Halima B. Maïnassara

Centre de Recherches Médicales et Sanitaires

634 Bd de la Nation YN034

BP 10887, Niamey, Niger

Tel. +22 720752 040; Fax +22 720753180

E-mail: halima@cermes.org
}

rence of 5 to 10 years. The toll can reach 1,000 cases for every 100,000 inhabitants leading to high morbidity and mortality rates despite the existence of effective and available antibiotics and vaccines against serogroups A and C. For the year 2003, the World Health Organization (WHO) estimated the morbidity due to meningitis in Africa to be between 394,000 and 497,000 cases, with 8,000 to 12,000 deaths (OMS, 2003). In 2005, the WHO reported mortality rates for $12 \mathrm{AMB}$ countries, based on notified, suspected cases that ranged from $4 \%$ in Mali to $26 \%$ in Benin (WHO, 2005). In Niger, the mortality rate was recently estimated at $15.7 \%$ based on laboratory-confirmed cases (Boisier et al., 2007). The way these epidemics spread in human populations at the local level has long puzzled public 
health workers. Environmental, economic, social, educational, cultural and genetic factors may have spatial distributions influencing the extent and intensity of a particular disease (Moore and Carpenter, 1999). The existence and variation of these factors could provide an explanation for the spatio-temporal variation in the distribution of the disease (Jia et al., 2009) and its persistence in the AMB populations. Spatio-temporal databases provided during routine surveillance may contain important information regarding disease distribution (Jepsen et al., 2009) and the geographical distribution of a disease can provide valuable clues helping us to understand how culture, environment and behaviour impact health (Moore and Carpenter, 1999). Indeed, any disease prone to outbreaks can be better understood by clarifying its occurrence in term of person, place and time (Greene et al., 2005). The objectives of this study were to explore the spatio-temporal patterns of meningococcal meningitis by:

(i) studying the evolution of annual clusters of the disease in Niger from January 2002 to June 2008; and

(ii) identifying areas with an increased risk of infection to assist epidemiologists in finding specific infection patterns.

\section{Materials and methods}

\section{Study area}

Niger is a vast and landlocked territory with an arid climate with a surface area of $1,267,000 \mathrm{~km}^{2}$ and a population estimated at 11 million in 2001 . From south to north, the country has three climate zones: the Sudanian, Sahelian and Saharan zones.

\section{Study design and data collection}

A routine prospective surveillance was performed in Niger from January 2002 to June 2008. Samples were collected as described previously (Boisier et al., 2007). Fluids were analysed using the polymerase chain reaction (PCR) method for Neisseria meningi- tidis, Streptococcus pneumoniae and Haemophilus influenzae (Sidikou et al., 2003; Chanteau et al., 2006b). Fresh cerebrospinal fluid (CSF) or samples taken using the trans-isolate method were examined by conventional bacteriology including Gram examination, culture and application of the latex agglutination test (Djibo et al., 2006). Some CSF samples were tested using a rapid diagnostic test (Chanteau et al., 2006a). Questionnaires accompanying the sampling were issued to collect some basic information such as the date the sample was taken, the health care institution, the city, the canton, the district, the age and sex of the patient.

The descriptive map of the meningitis incidence rate by department was edited using the SAS programme, version 9.1 and based on the geographical coordinates of Niger as obtained at the following web site: http://www.maplibrary.org/. For the spatio-temporal analysis, the geographical coordinates of cantons and villages were obtained from the freeware Google Earth, version 5.0, available at: http://earth.google.fr/ and converted into $\mathrm{x}$ - and $\mathrm{y}$ coordinates (projection in two dimensions) using the Convers software, version 2.9, available at: http://vtopo.free.fr/. The border coordinates for Niger were obtained in UTM32 format at: http://www.maplibrary.org/stacks/Africa/Niger/, and then imported into $\mathrm{R}$, version 2.7.0.

National, regional, departmental and cantonal data by age group were obtained from the 2001 population census available at the website of the Institut National de la Statistique (INS) (http://www.stat-niger.org/). The number of inhabitants per village was obtained directly from the INS as given by Repertoire National des Communes (RENACOM).

\section{Case definition}

A suspected case is essentially characterised by clinical symptoms. Depending on the age of the patient and clinical signs, three definitions of meningitis in Africa have been provided by WHO (WHO, 1998). For adults and children over the age 
of 1 year, the definition is the presence of fever of $38.5^{\circ} \mathrm{C}$ or higher combined with neck muscle tension and petechiae or purpura. In children under the age of 1 year, the definition is simply presence of fever and a bulging fontanel. A likely case is defined as a suspected case with cloudy CSF, or a suspected case in an epidemic context. A clear case, finally, is confirmed when the etiological diagnosis is biologically determined based on a CSF sample taken from a suspected or likely case.

\section{Spatio-temporal analysis}

In order to account for the seasonality of meningococcal meningitis, the analysis was conducted according to the epidemiological year, beginning on 1 July of one year and ending on 30 June of the following year. The spatial description of cases was carried out at the village level. However, as we did not know the exact census for all villages of Niger, we were unable to test the significance of clusters at this level. Therefore, the patient's home canton was chosen as the spatial unit and the date on which the CSF sample was taken as the temporal unit. The p-value was considered significant at a level of $<0.05$.

Cumulative incidence rates were calculated for every 100,000 inhabitants and graphed by department using SAS, version 9.1. A correlation between the meningitis incidence rate at the department level and the population density was then sought using the Pearson correlation coefficient. The spatial descriptive analysis at the village level was done using R, version 2.7.0. Poisson's model was used with the SaTScan software, version 5.1, available at: http://www.satscan.org, to search for spatial clusters at the canton level (Kulldorf, 1997; Kulldorf and Information Managements Services, 2004). The Kulldorff scan method allowed us to locate and detect spatial and/or temporal aggregates while taking into account the temporal and spatial variations of the population at risk. Under the null hypothesis, the number of expected cases is proportional to the population size, while the alternative hypothesis requires that the rate of cases is higher within the window than outside. Under Poisson's hypothesis, the likelihood function for a given window is proportional to:

$$
\left(\frac{c}{c_{e}}\right)^{c}\left(\frac{C-c}{C-c_{e}}\right)^{C-c} I(f),
$$

where $C$ is the number of cases, and $c_{e}$ the number of expected cases in the window under the hypothesis of uniform distribution. $I(f)$ is an indicative function that is equal to 1 when the window $f$ has more cases than the expected number under the null hypothesis, and 0 otherwise. A cluster is a number of health events located close together in space and/or in time (Moore and Carpenter, 1999).

\section{Spatial and temporal evolution}

A Kolmogorov-Smirnov test was used to compare the distribution of case occurrence from one year to another. Annual incidence rates were calculated for every 100,000 inhabitants by department using Microsoft Excel, 2003 version. Finally, the evolution of meningococcal meningitis dynamics was studied in terms of both time and space by groups of 15 at the canton level.

\section{Results}

During the six and a half year surveillance period, the Centre de Recherches Médicales et Sanitaires (CERMES) identified 3,979 CSF samples testing positive for $N$. meningitidis. The average age was 9.2 years (distance-type $=7.3$ ). The sex ratio was 1.42 in favour of males. The cumulative incidence varied from $<3$ cases for every 100,000 inhabitants in the northern departments to 123 cases for every 100,000 inhabitants in the department of Birni N'Konni (Fig. 1). The incidence was generally higher in the south-east, such as in the departments of Madarounfa (97.63 for every 100,000 inhabitants), Aguié (87.75 for every 100,000 inhabitants), Matameye (69.37 for every 100,000 inhabitants) and Magaria (55.75 for every 100,000 inhabitants). 


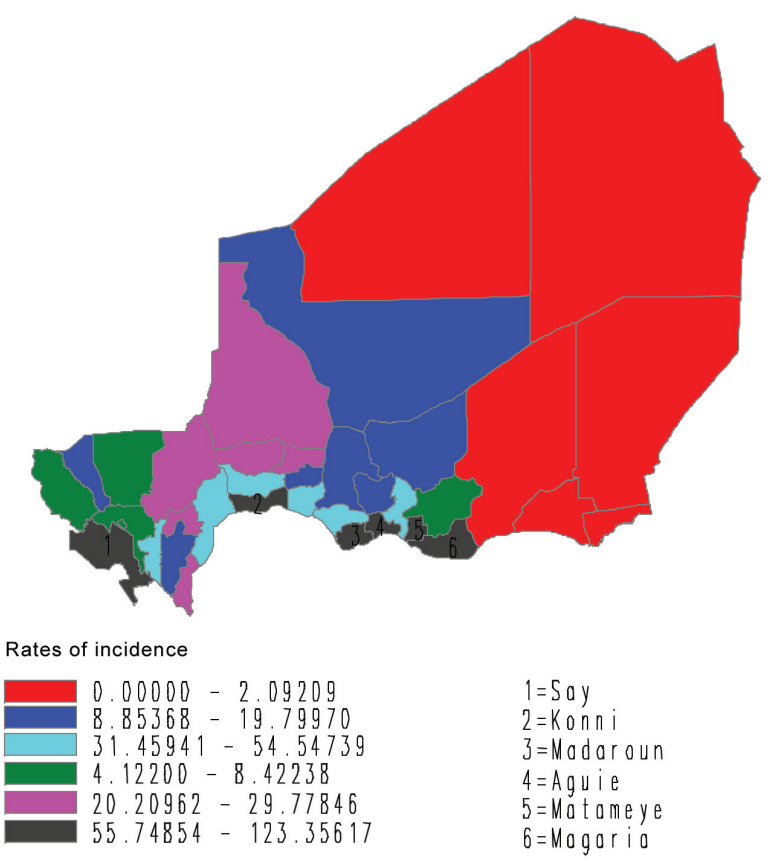

Fig. 1. Cumulative incidence for the period 2002 to 2008 of meningococcal meningitis per 100,000 inhabitants in Niger, calculated by department and based on the 2001 population figures.

This was also the case for the western part of the country, such as the department of Say (68.83 for every 100,000 inhabitants). Pearson's correlation coefficient for cumulative incidence and population density (number of inhabitants per $\mathrm{km}^{2}$ ) by department was positive, but weak and not significantly different from $0(\mathrm{r}=0.20 ; \mathrm{P}=0.24)$. Figure 2 depicts the distribution of meningococcal meningitis at the village level between July 2002 and June 2008.

\section{Spatial clusters}

To account for the size of the population at risk, a cluster study was carried out. In an area that included 34 cantons the SatScan analysis identified a likely cluster of 558 cases at a high level of significance in the 2002-2003 epidemiological year, which represented $70.5 \%$ of all cases of that period (relative risk $(\mathrm{RR})=7.85 ; \mathrm{P}=0.001)$. In the same year, a secondary cluster of 70 cases was found $(R R=3.05$; $P$
$=0.001)$ in the capital. Other statistically significant clusters were identified in the following years and many of them reappeared annually, approximately in the same places. A total of 15 clusters were detected during the whole study period, i.e. from July 2002 to June 2008 (Fig. 3).

\section{Spatio-temporal evolution}

The distribution of meningococcal meningitis cases, occurring over the course of the year in 2002, 2005 and 2007, shows an initial peak between 12 November and 1 January. A second, more significant peak arose each year around the $100^{\text {th }}$ day, which corresponds to mid-April, specifically around 10 April (Fig. 4). All curves drop off by the end of May or at the beginning of June. The case occurrence distribution functions varied greatly from year to year ( $\mathrm{P}<0.001)$. Year-by-year density comparisons, done for all combinations of years, showed pvalues ranging from 0.036 for 2005 and 2007, to $<0.001$ for 2003 and 2008, 2005 and 2008, 2006 and 2008, and finally 2007 and 2008.

The first cases of meningococcal meningitis in the 2002-2003 epidemiological year appeared in the south and south-west; the next year they appeared in the south-west and the south-east. In 2004-2005, the area in which the first cases appeared widened towards the centre of Niger in addition to the usual areas. The first cases became more frequent in the south-eastern communes and less frequent in the south-western communes. Afterwards, the first cases appeared particularly in the south-east. Overall, most first cases occurred in the south-eastern communes. The dynamic evolution of a group of 15 cases (shown in video format in the online version of this paper), shows an increase in the number of cases in the cantons in the central and southwestern parts of the country followed by a higher concentration in the south-east. In 2005-2006, the case occurrences first spread from the south-east towards the southwest and then moved towards the centre. The last cases, however, did not have any particular spatial tendency. 

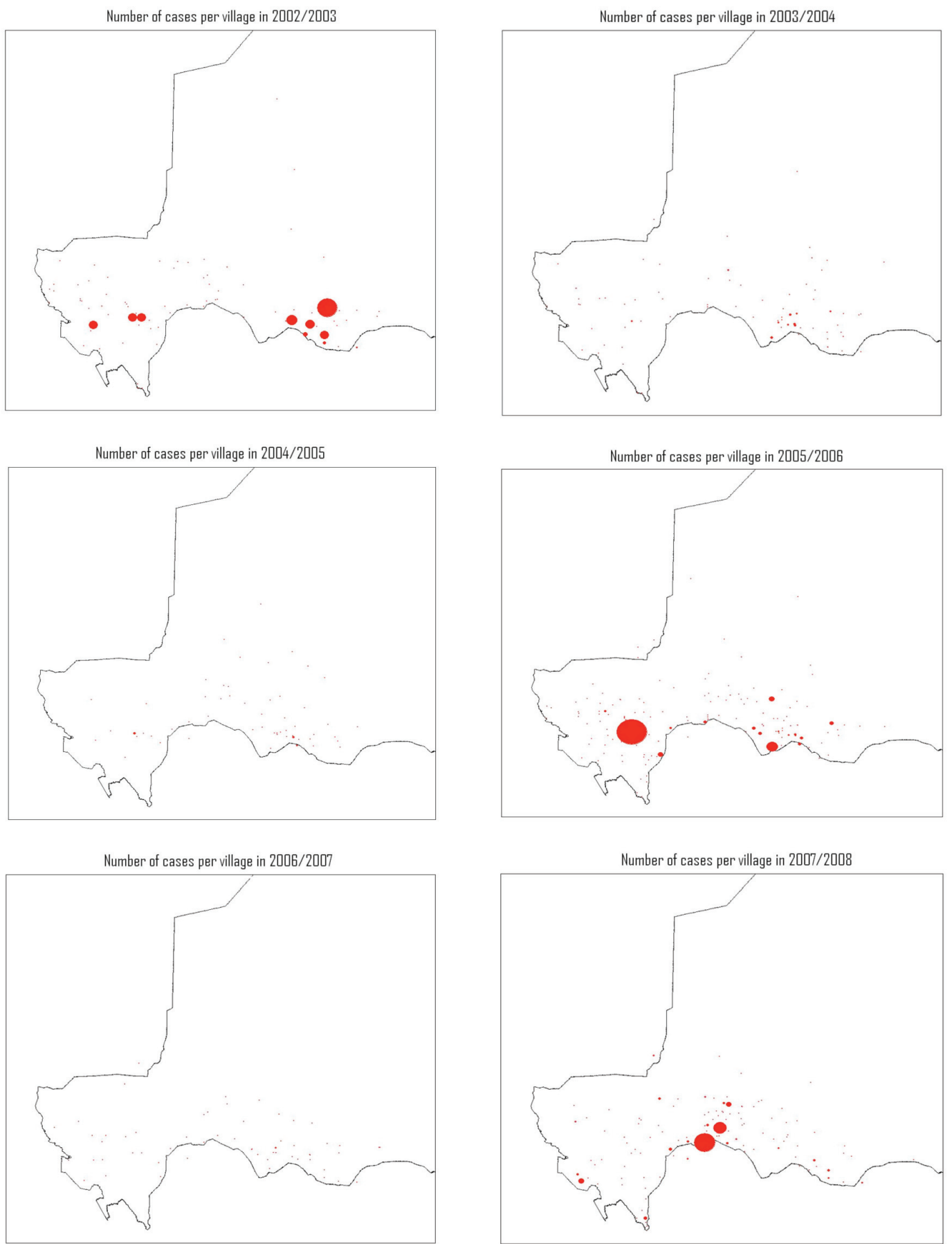

Fig. 2. Representation of the annual meningococcal meningitis cases at the village level for the period July 2002 to June 2008 in Niger. The red points represent the patients' villages and the size of the points is proportional to the number of cases of meningitis in the village in question. 

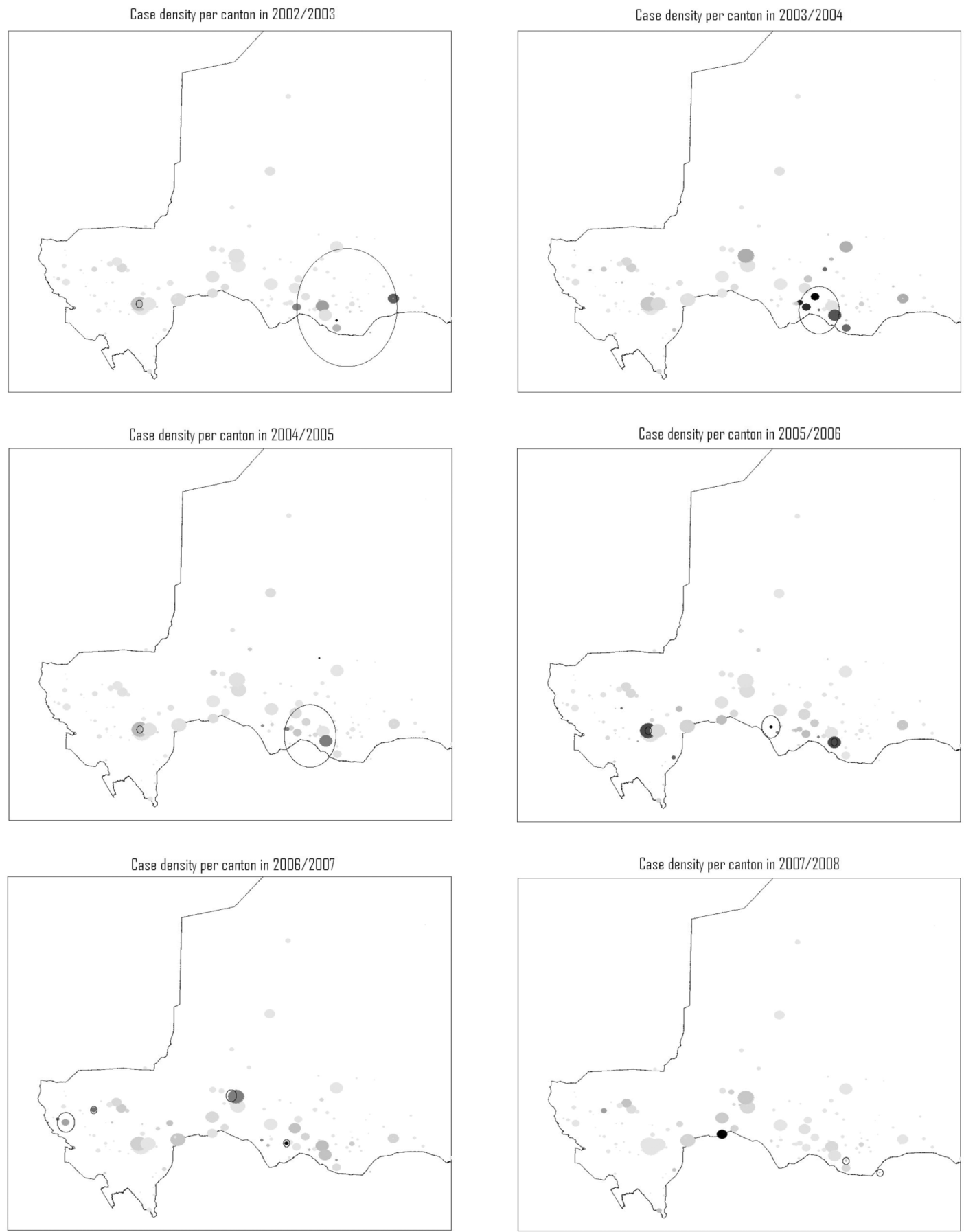

Fig. 3. Representation of the annual meningococcal meningitis case density and spatial clusters for the period July 2002 to June 2008 in Niger. The density of cases by canton is shown as grey points, the size of which is proportional to the size of the canton population. The grey scale is proportional to the density of cases, i.e. the darker the point, the higher the density. Statistically significant clusters are outlined by circles. 


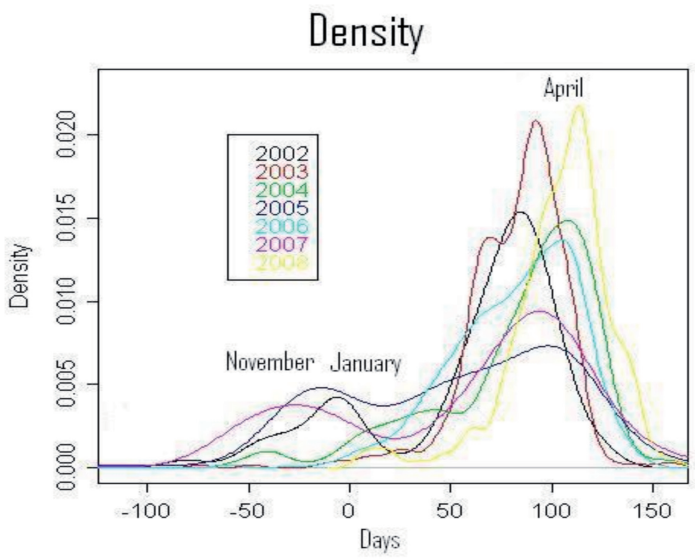

Fig. 4. Distribution of meningococcal meningitis case occurrences in Niger over the period July 2002 to June 2008.

\section{Discussion}

To our knowledge, we report the first study aiming to examining the spatio-temporal tendencies of meningococcal meningitis cases at the canton level (average number of inhabitants $=85,000$ ) as spatial unit and the day as temporal unit. This scale of investigation was made possible by the following technical assets: the free availability of SaTScan software and Google Earth, a good monitoring system, and the availability of data on the time and place of case occurrence, demographics and laboratory techniques for the three infectious agents. As pointed out by Hasting et al. (1997), an international agreement on methodology would be useful as it is difficult to compare clusters from different studies because of the differences in case and cluster definition. Indeed, one of the reasons for choosing SaTScan for this study is that it is the most widely used software package for cluster analysis (Elias et al., 2006), increasing the chance to be able to compare the results with those of future analyses.

Fifteen statistically significant spatial clusters of meningitis were found in six epidemiological years with a high number of cases (maximum $=558$ cases; minimum $=9$ cases). In Germany, the number of significant clusters of meningococcal disease identified from December 2001 to June 2005 was 26, with an average of 2.6 patients (Elias et al., 2006). Approximately 114 clusters occurred in England between 1995 and 2001, in schools and nursery schools, $64 \%$ of which contained two or more confirmed cases (Davison et al., 2004).

The clusters were more frequent on the Nigerian border in the south-eastern part of the country. The high numbers of people that cross the border in this part of the country for economic, family and cultural reasons could explain this situation. Given that the border with Nigeria is more imaginary than real, the area densely populated and people highly mobile, meningitis can easily be passed from one country to another. Indeed, the risk of cluster occurrence was found to be higher for the cantons of Konni and Tibiri-Guidan Roumdji, which are located at the Nigerian border. Free access to meningococcal disease care could be the reason for Nigerian patients attending Niger's health care structures. When taking into account only cases occurring in Niger, we were not able to avoid this issue in the spatial analysis. However, this argument alone does not explain the spatial variability, because cantons located in the southernmost point of the country do not experience as many cases as the cantons of the south-east although they also have much contact with Nigeria. However, there are enormous ethnic and cultural differences between the west and the east. According to the INS, the fertility level of the Djerma-Sonraï and Gourmantché linguistic/social groups living in the west and the south-west of the country reportedly decreased between 1988 and 2001, contrary to that of the Hausa living in the centre and south-eastern part of the country (http://www.stat-niger.org/).

Vaccination status could also have influenced the occurrence of cases and clusters in this area. The frequency of aggregates in the south-east was not linked when the analysis accounted for regional age distribution (data not shown).

This study probably did not cover the entire population of Niger affected by meningococcal meningitis. We recognise that the situation could vary within departments or regions due to unequal 
availability of human and financial resources and difficulties to provide CSF samples at the central level. Further reasons include geographical remoteness, which has an impact on the lumbar puncture procedure, and the different concerns and unequal efforts put forth by the staff aimed at detecting suspected cases. Based on these reports, it is possible that a selection method could have impacted our results, particularly in terms of the incidence rates. Calculations based on confirmed cases probably underestimated the incidence because CSF samples were not available for all cases, and some of them (although fewer) were not possible to examine due to insufficient quantity or pronounced purulence of the fluid. Furthermore, some patients afflicted with full-blown bacterial meningitis could not attend health care centres in time, while other patients did not show up due to economic or cultural reasons (e.g. use of traditional medicine), thus resulting in under-reporting. The incidence calculations were based on demographic data taken from the 2001 population census. The estimates did therefore not take into account possible variations in the population over time, a fact difficult to approximate. Since each patient's residency was defined spatially at the canton level, the exact trajectory of transmission was not able to be followed. We were also unable to draw any conclusions regarding individual risk factors. Since our statistical methods used the barycentre of the main urban area for every canton, the extent of the aggregates was probably overestimated due to imprecise geographical coordinates. However, in the absence of a more precise alternative, the choice of the cantonal unit can be considered as an asset, since transmission could occur in places other than the residence (e.g. schools, markets and workplaces). Because these places are located in the same area as the patients' homes, this factor did not credibly affect our results.

The spatial aggregate identification performed in this study should be a guide for more advanced epidemiological research in the future, aiming to identify the local, socio-demographic, cultural, and espe- cially environmental influences on the transmission agents of meningococcal meningitis. In fact, for risk factor analysis, particularly in an exploratory way, geographic variables should be used to identify descriptive relationships, as a basis for researching causal relationships (Moore and Carpenter, 1999). Vaccine prevention should be focused more in the south-east and be implemented in cooperation with Nigeria, the large neighbour to the south. In the absence of specific options for the prevention of epidemics, the inclusion of data about time and place of diseases occurrence, prospectively in a spatiotemporal information system could support monitoring activities and make possible to use limited resources to serve the most vulnerable areas. Given the onerous burden of infectious diseases in developing countries with weak economic resources, accessible epidemiological surveillance systems should be introduced to help quickly identifying regions at high risk of epidemic (Reinhardt et al., 2008). Clinicians would thus be informed and better equipped to assist districts in the face of an epidemic. In fact, the tools of the spatio-temporal information system are powerful and able to rapidly examine spatial patterns. The development of geographical information systems (GIS) achieved over the past 20 years has created a faster and more powerful means of examining spatial patterns (Moore and Carpenter, 1999). Consequently, they offer an opportunity to endorse public health initiatives for meningococcal meningitis and other diseases, possibly even the institution of an early-warning system.

In conclusion, high-risk areas with statistically significant spatio-temporal patterns were identified in Niger, particularly in the south-eastern part of the country, which could be useful in establishing hypotheses for future prospective studies on epidemic tendencies and empirical risk factors in the AMB.

\section{Acknowledgements}

We are grateful to Jean-François Jusot for providing helpful comments and for critically reading out manuscript. We wish 
to thank the CERMES meningitis group (Pascal Boisier, Suzanne Chanteau, Jean-Marc Collard, Amina Amadou, Fati Sidikou, Bassira Boubacar Issaka, Ali Elhadj, Amadou Moussa, Sacou Djibo, Ramatou Yahaya, Ali Sidiki, Tohon Zilahatou, Jean-François Jusot and Jocelyne Rocourt), Unité des Neisseria, Institut Pasteur, Paris, France; the WHO Collaborating Centre for Reference and Research on Meningococci-IMTSSA, le Pharo Marseille, France for scientific and technical support, nurses and doctors of Niger for taking fluid samples. Financial support: Sanofi Pasteur, Institut Pasteur, MAE and the WHO.

\section{References}

Boisier P, Maïnassara HB, Sidikou F, Djibo S, Kairo KK, Chanteau S, 2007. Case-fatality ratio of bacterial meningitis in the African meningitis belt: we can do better. Vaccine 25, 24-29.

Chanteau S, Dartevelle S, Mahamane AE, Djibo S, Boisier P, Nato F, 2006a. New rapid diagnostic tests for Neisseria meningitidis serogroups A, W135, C, and Y. PLoS Med 3, e337.

Chanteau S, Sidikou F, Djibo S, Moussa A, Mindadou H Boisier P, 2006b. Scaling up of PCR-based surveillance of bacterial meningitis in the African meningitis belt: indisputable benefits of multiplex PCR assay in Niger. Trans R Soc Trop Med Hyg 100, 677-680.

Davison KL, Andrews N, White JM, Ramsay ME, Crowcroft NS, Rushdy AA, Kaczmarski EB, Monk PN, Stuart JM, 2004. Clusters of meningococcal disease in school and preschool settings in England and Wales: what is the risk? Arch Dis Child 89, 256-260.

Djibo S, Njanpop Lafourcade BM, Boisier P, Moussa A, Kobo G, Sidikou F, Hien A, Bieboure G, Aguilera JF, Parent du Chatelet I, Gessner BD, Chanteau S, 2006. Evaluation of the Pastorex meningitis kit for the rapid identification of Neisseria meningitidis serogroups A and W135. Trans R Soc Trop Med Hyg 100, 573-578.

Elias J, Harmsen D, Claus H, Hellenbrand W, Frosch M, Vogel U, 2006. Spatio-temporal analysis of invasive meningococcal disease, Germany. Emerg Infect Dis 12,
1689-1695.

Greene SK, Schmidt MA, Stobierski MG, Wilson ML, 2005. Spatio-temporal pattern of viral meningitis in Michigan, 1993-2001. J Geogr Syst 7, 85-99.

Hastings L, Stuart J, Andrews N, Begg N, 1997. A retrospective survey of clusters of meningococcal disease in England and Wales, 1993 to 1995: estimated risks of further cases in household and educational settings. Comm Dis Rep 7, 195-200.

Jepsen MR, Simonsen J, Ethelberg S, 2009. Spatio-temporal cluster analysis of the incidence of campylobacter cases and patients with general diarrhoea in a Danish country, 19952004. Int J Health Geogr 8, 11.

Jia ZW, Li XW, Wang W, Cheng SM, 2009. Promising of spatial-temporal model in public health. Chin Med J 122, 349350 .

Kulldorff M, 1997. A spatial scan statistic. Commun Stat Theory Methods 26, 1481-1496.

Kulldorff M and Information Managements Services, 2004. SatScan v5.1: software for spatial and space-time scan statistics. http://www.satscan. org (accessed: 12 May 2009).

Lapeyssonnie L, 1963. Cerebrospinal meningitis in Africa. Bull World Health Organ 28, 1-114.

Moore DA, Carpenter TE, 1999. Spatial analytical methods and geographic information systems: use in health research and epidemiology. Epidemiol Rev 21, 143-161.

OMS, 2003. Rapport sur la santé dans le monde. Façonner l'avenir WHO, Geneva, Switzerland.

Reinhardt M, Elias J, Albert J, Frosch M, Harmsen D, Vogel U, 2008. EpiScanGIS: an online geographic surveillance system for meningococcal disease. Int J Health Geogr 7, 33.

Sidikou F, Djibo S, Taha MK, Alonso JM, Djibo A, Kairo KK, Chanteau S, Boisier P, 2003. Polymerase chain reaction assay and bacterial meningitis surveillance in remote areas, Niger. Emerg Infect Dis 9, 1486-1488.

WHO, 1998. Control of epidemics meningococcal disease. WHO Practical Guidelines, 2nd edn. Document WHO/EMC/ BAC98.3. WHO, Geneva, Switzerland.

WHO, 2005. Enhanced surveillance of epidemic meningococcal meningitis in Africa: a 3-year experiment. Wkly Epidemiol Rec 80, 313-320. 